\title{
Enhancement of Equal Gain Combiner for Detection of Spectrum Hole in a Cognitive Radio System
}

\author{
Ojo S. I. ${ }^{1}$, Adeyemo Z. K. ${ }^{1, *}$, Ojo F. K. ${ }^{1}$, Adedeji A. A ${ }^{2}$, Semire F. A. ${ }^{1}$ \\ ${ }^{1}$ Department of Electronic and Electrical Engineering Ladoke Akintola University of Technology Ogbomoso, Nigeria \\ ${ }^{2}$ Department of Mechatronics Engineering, Osun State College of Technology Esa Oke, Nigeria
}

Received July 16, 2020; Revised September 2, 2020; Accepted September 17, 2020

\section{Cite This Paper in the following Citation Styles}

(a): [1] Ojo S. I., Adeyemo Z. K., Ojo F. K., Adedeji A. A, Semire F. A. , "Enhancement of Equal Gain Combiner for Detection of Spectrum Hole in a Cognitive Radio System," Universal Journal of Electrical and Electronic Engineering, Vol. 7, No. 5, pp. 289 - 298, 2020. DOI: 10.13189/ujeee.2020.070503.

(b): Ojo S. I., Adeyemo Z. K., Ojo F. K., Adedeji A. A, Semire F. A. (2020). Enhancement of Equal Gain Combiner for Detection of Spectrum Hole in a Cognitive Radio System. Universal Journal of Electrical and Electronic Engineering, 7(5), 289 - 298. DOI: 10.13189/ujeee.2020.070503.

Copyright $\bigcirc 2020$ by authors, all rights reserved. Authors agree that this article remains permanently open access under the terms of the Creative Commons Attribution License 4.0 International License

\begin{abstract}
Cognitive Radio System (CRS) is of paramount importance in wireless communication for spectrum management. However, channel impairment affects accurate Spectrum Hole Detection (SHD) in CRS resulting in harmful interference to a licensed user. Conventional Equal Gain Combiner (EGC) which is one of the techniques being used to solve the problem suffers from hardware complexity resulting in high Sensing Time (ST). Hence, in this paper, an Enhanced EGC (EEGC) for SHD in a CRS is proposed to reduce the hardware complexity of conventional EGC. Ten thousand $(10,000)$ bits are randomly generated, modulated using Binary Phase Shift Keying (BPSK) scheme and propagated over Rayleigh fading channel. The received signals through three antennas are scanned by three Selection Combiners (SCs) to select the branch with highest Signal to Noise Ratio (SNR). The outputs of the three SCs are then combined using conventional EGC. The output of conventional EGC is used as input to single Energy Detector (ED) to determine the energy of the combined signal. A mathematical expression for Probability of False Alarm (PFA) for the proposed technique is derived using $X^{2}$ distribution to set thresholds at 0.01 and 0.02 . The proposed technique is simulated using MATLAB software and evaluated using Probability of Detection (PD), ST and Probability of Missing (PM). The results obtained show that EEGC gives better performance with lower ST, higher PD and lower PM.
\end{abstract}

Keywords Equal Gain Combiner, Probability of
Detection, Licensed User, Probability of Missing, Spectrum Hole, Cognitive Radio, Unlicensed User, Energy Detector

\section{Introduction}

Wireless Communication (WC) is the exchange of information between two or among many points through an unbounded environment. In WC, information is transmitted through the air using electromagnetic waves. The exponential rise in the number of users accessing WC services resulting in rapid increase in the demand for high data rate services leads to spectrum scarcity. Previous researches have shown that spectrum scarcity is not only limited to insufficient spectrum but also to poor utilization of the allocated spectrum. In 1999, Mitola proposed a technique known as Cognitive Radio Networks (CRNs) that overcome spectrum scarcity $[1,2]$. Cognitive Radio (CR) is a WC technique in which a transceiver intelligently senses the spectral environment over a wide frequency band to detect unused spectrum and provide wireless links through the unused spectrum [3, 4]. CR is made up of two users namely: licensed Users (LUs) and Unlicensed Users (ULUs). LUs are authorized users who have the precedence to use the assigned spectrum, while ULUs are unauthorized users who are allowed to make use of assigned spectrum only when LU is not present $[5,6,7,8]$. 
Spectrum Sensing (SS) is the process of determining the idle spectrum (spectrum hole) in a CR network. It is one of the key operations in $\mathrm{CR}$ system to evaluate the performance of CR $[9,10,11]$. The accuracy of spectrum hole detection, which is the ability of ULU to sense LU without interference depends on the channel impairments. The channel impairment such as atmospheric factors, different type of obstacles affects signal propagation resulting in fluctuation of the received signals causing poor reception of the signal at ULU. The poor signals reception of the ULU makes the accurate spectrum detection to be difficult and results in hidden licensed user which poses to be one of challenges of CRS [12]. The problem of hidden licensed user is overcome by the use of multiple antennas at the ULU that receives multiple copies of LU signal. However, the performance of multiple antenna technique in CRS depends on diversity combiner and detector employed. Energy Detector (ED) and Eigenvalue Detector (EVD) are the two major detectors used in CRS to detect the presence of spectrum hole. The choice of these detectors is due to fact that no prior information about $\mathrm{LU}$ signal is required, therefore, synchronization is not necessary. ED is very simple to implement and has a better detection rate in comparison with EVD. Therefore, this leads to adoption of ED to determine the presence of spectrum hole in a CRS $[13,14]$. Several techniques being proposed to take care of the hidden LU problem in CRS include Equal Gain Combiner (EGC), Selection Combiner (SC) and Square Law Combiner (SLC). From the existing literature, EGC and SLC have better performances with high detection rate even at a low signal strength than SC but at the expense of Hardware Complexity (HC) [15]. SC is often used in practice, but there is a trade-off between the $\mathrm{HC}$ and the detection rate when compared with SLC and
EGC [16]. Therefore, in this paper, an Enhanced EGC (EEGC) is proposed to improve the detection rate and at the same time reduce the hardware complexity of the conventional EGC.

\section{Equal Gain Combiner with Energy Detector}

One of the techniques previously used to solve the problem of hidden LU in a CRS is Equal Gain Combiner (EGC) with ED. EGC is a combining technique in which all the branches of the signals weighed with the same weight before summing and co-phased to increase signal strength. In EGC, the received signals from different branches are first multiplied with equal weights and then co-phased to avoid signal reduction before summing up. In detecting SH, output of EGC is used as input to Energy Detector (ED) in determining the energy of the received LU signals. The energy obtained is, then, compared with set threshold to find the presence of SH. EGC with ED is usually employed to find spectrum hole due to high detection rate, but characterized with hardware complexity due to multiple RF chains and Matched Filters (MFs). The received signal passes through each of the RF chains, MFs and then, sums all the signals before applying ED as shown in Fig. $1[10,17]$. The output signal of EGC with ED ' $E_{E G C}$ ' is given by [17] as

$$
E_{E G C}=\sum_{K=1}^{N}\left|\left(\sum_{j=1}^{N_{r}} q_{j}(k)\right)\right|^{2} .
$$

where: $\mathrm{N}$ is the baud length

$N_{r}$ is the number of antennas,

$q_{j}(k)$ is the LU transmitting signal

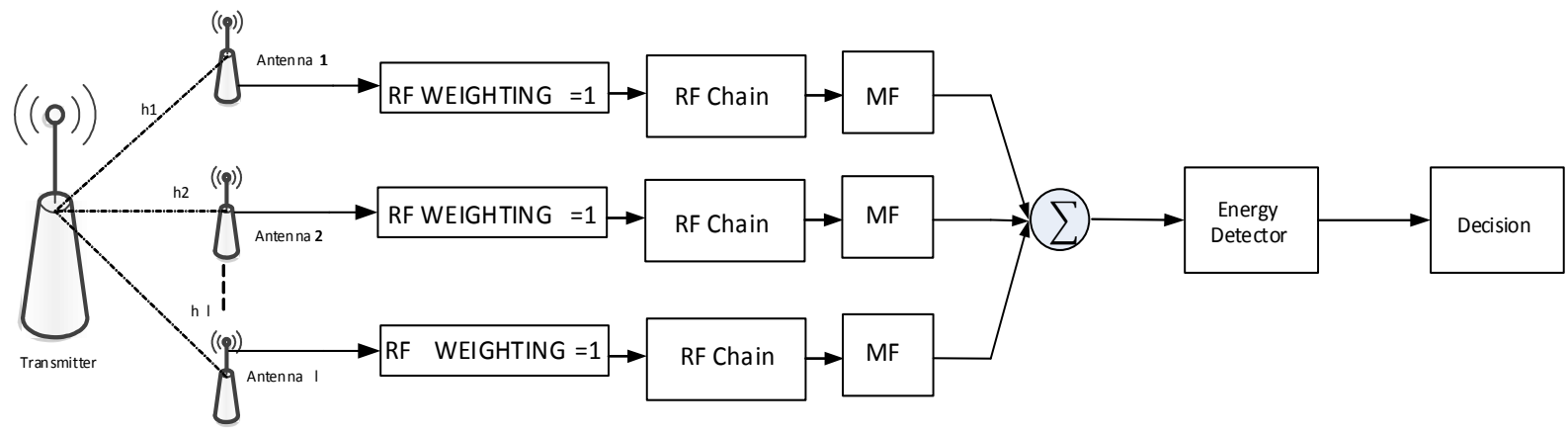

Source: $[10,17]$

Figure 1. Equal Gain Combiner with ED 


\section{Related Work}

In [18] EGC for SS in Cognitive Radio systems was proposed to solve hidden LU problem in CR networks. The technique comprised five ULU antennas that received LU signal and multiplied with equal branch weight. The resultant signal was then made to pass through RF chain and Match Filter (MF) before applying ED to obtain the energy of the received signals. Output of ED was then compared with the set threshold to make a decision on the presence of $\mathrm{SH}$. The result of the paper revealed that, the technique had high detection rate but at the expense of $\mathrm{HC}$. Also, in [11] ED based SS over fading channels was proposed. LU interference in CR was addressed using SC method. In the paper, ULU selected the path with highest signal strength and received signal from that path. The received signal was applied to ED to get energy of the selected signal and make decision on presence of SH. The results of the paper showed a reduction in $\mathrm{HC}$ but at the expense of low detection rate. In [19], a multi-node spectrum sensing based on ED for dynamic spectrum access was proposed to combat the problem of PU interference in $\mathrm{CR}$ using Collaborative-Technique Hard-Decision (CTHD) with logic table. In the paper, each ED received signal from each antenna and made a decision on the redundancy of the spectrum. The decision from individual ED was then combined to make a final decision using logical table. The result of [19] showed that the probability of missing was low but with high sensing time due to several energy detectors involved in the technique.

Multiple detectors based analytical performance of SS is presented in [20] to address hidden LU problem in CRS using Maximal Ratio Combiner (MRC) technique. The received signal from different branches were multiplied with the channel gain and summed before applying single ED to get the energy of the combined signal. The obtained energy was then compared with the set threshold to determine the redundancy of spectrum. The result of [20] showed that, the technique had a higher detection rate but suffered from hardware complexity due to estimation of channel gain on each branch thereby leading to high sensing time. Also, [21] worked on ED of unknown signal over fading channel to address hidden LU problem in a CRS using SLC method. In [21], five antennas, five EDs and a combiner were used to determine the presence of LU signal. ED received signal from each of the antennas and output of each of the EDs were combined and compared with the set threshold to make a decision on the presence of spectrum hole. The result of [21] showed that, the technique had a high detection rate even at a very low signal strength. Also, it was very easy to set decision threshold as it did not require perfect channel knowledge but with $\mathrm{HC}$ as a result of several EDs leading to high sensing time and high implementation cost. Past researches have shown that EGC, CTHD, MRC and SLC have better performances but suffer from HC. while, SC was associated with a poor detection rate. Therefore, this work proposes an Enhanced EGC (EEGC) technique to reduce the $\mathrm{HC}$ of existing EGC and fluctuation of the received signal.

\section{Materials and Method}

\section{A. Spectrum Sensing in Cognitive Radio}

Signal detection technique which is used to describe the method that reveals the presence of SH known as SS in a noisy environment is employed in this work. The technique is formalized as two hypothesis tests $H 1$ and $H 0$ which represent the presence and absence of LU, respectively. $H 1$ and $\mathrm{HO}$ indicate that $\mathrm{LU}$ communicates and $\mathrm{LU}$ does not communicate, respectively. The two hypotheses are given by $[22,23,24]$ as.

$$
\begin{gathered}
H_{1}: M(n)=S(n) h+W(n) \\
H_{0}: M(n)=W(n)
\end{gathered}
$$

where: $M(n)$ is the received signal by ULU

$S(n)$ is the LU transmitting signal

$h$ is the channel gain

$W(n)$ is AWGN present

From Equations (2) and (3), four different possible cases that can occur in signal detection are defined: firstly, Probability of Detection (PD) which is declaring $H 1$ when LU signal is actually present and Secondly, Probability of Missing (PM) which describes declaring idleness of spectrum when LU signal is present. Probability of False Alarm (PFA) is the third one that describes the occupancy of the spectrum when LU is not actually present and the fourth one is declaring $H_{0}$ when LU is not present. PM is the biggest problem in spectrum sensing because increase in PM, increases the chances of ULU interfering with the LU. However, it is required to have PFA as low as possible in order for the system to dynamically make use of the available idle spectrum [25].

\section{B. Energy Detector in Signal Detection}

Energy Detector (ED) is used in signal detection to find the spectrum hole based on the energy of the received LU signal. It is a commonly used detector due to its low computational complexity and can be implemented in time domain as well as frequency domain. The detection is carried out by comparing the energy of the LU signal with the set threshold. The set threshold is a function of PFA [24]. This detector has a Band-Pass Filter (BPF) which allows certain frequency range called the passband to pass while attenuating the others, a squaring device and an integrator to obtain the energy of the received signal. The obtained energy at the output of the integrator is then compared with the set threshold and if the obtained energy is above the threshold, then, spectrum is occupied, that is, spectrum hole is not exist otherwise spectrum is idle [14, 
21, 26]. ED approach is implemented without any prior knowledge of LU signal, but has some challenges which include poor performance under low SNR [26]. The output of $E D, " E "$ is given by [27] as

$$
\begin{gathered}
E=\sum_{n=1}^{N}|S(n)|^{2} \\
E \geq \gamma .
\end{gathered}
$$

where: $\gamma$ is the set threshold

Equation (5) represents the threshold which indicates whether PU signal exists or not. Small noise power, long detection time and high signal power resulting in better performance of ED [24].

\section{Selection Combiner}

The choice of Selection Combiner (SC) is made due to its low $\mathrm{HC}$ and ability to choose the signal on the branch with the highest SNR. In this combiner, the sum of the individual signal from different branches is not required since output of SC is equal to the signal on only one branch $[28,29,30,31]$. It is one of the simplest detection techniques in which ULU is assumed to have the knowledge of channel status at a particular time and thus, selects the branch with highest gain and receives signal from that branch. The SNR output of the SC ' $\gamma$ ' is given by $[20,32,33,34]$ as

$$
\gamma=\max \left\{\gamma_{1} \ldots \ldots \ldots \gamma_{L}\right\}
$$

where: $\gamma_{L}$ is the received SNR per bit of the $l^{\text {th }}$ branch at any instant

\section{Rayleigh Distribution}

Rayleigh fading distribution is adopted due to its appropriate distribution that matches a cluster environment in a CR. It is a statistical distribution used for modeling the effect of signals propagating in a cluster environment where the effect of heavily built up environments is of paramount importance. It is mostly useful when there is no dominant line of sight along the propagation channel. In this environment, the received signal characterized by obstructions is the summation of all the diffused signal components. The amplitude of the received signal is described by Rayleigh Probability Density Function (PDF).
The PDF of this distribution denoted by $P_{R}(r)$ is given by $[28,32]$ as

$$
P_{R}(r)=\frac{r}{\sigma^{2}} \exp -\left(\frac{r^{2}}{2 \sigma^{2}}\right) \quad 0<r<\infty .
$$

where: $r$ is the magnitude of the signal received $\sigma$ is the peak value of the signal received $\sigma^{2}$ is the average power of the signal received

\section{E. Development of an enhanced EGC using SC}

The multiple copies of LU signals propagated through the Rayleigh fading channel considered are received by ULU multiple antennas and first scanned by SC. Scanning through the received multiple antenna signals is carried out to select the path with the highest value of SNR and ignore the weaker branches through SC. There are nine branches at the inputs of three SCs which result into three branches at the SCs output, through branch selection based on highest SNR. The three branches at the output of SCs are used as inputs to EGC. The three branches of signal are then co-phased, weighted, and summed up. Output of EGC is then used as input to ED to determine the energy of the received LU signal. The obtained energy is compared with the set threshold to decide on the presence of SH. The assumption is that if the values of energy obtained are above the set threshold, $\mathrm{SH}$ is not present due to ongoing transmission of LU, otherwise, spectrum hole is present, that is, the spectrum is idle. The threshold is set using PFAs of 0.01 and 0.02 . Block diagrams of the proposed technique are shown in Figs. 1 and 2, where $h$ represents Rayleigh fading channel. MF and RF chain are the major components that determine the complexity of EGC. However, the number of branches to combine are proportional to number of RF chains and MFs in conventional EGC. In this paper, nine branches were considered using conventional EGC as a combiner requires nine MFs and nine RF chains leading to hardware complexity. The number of RF chains and MFs are reduced in this paper from nine (9) to three (3) using SC to select only the best three paths and ignore weaker ones, thereby reducing the $\mathrm{HC}$. 


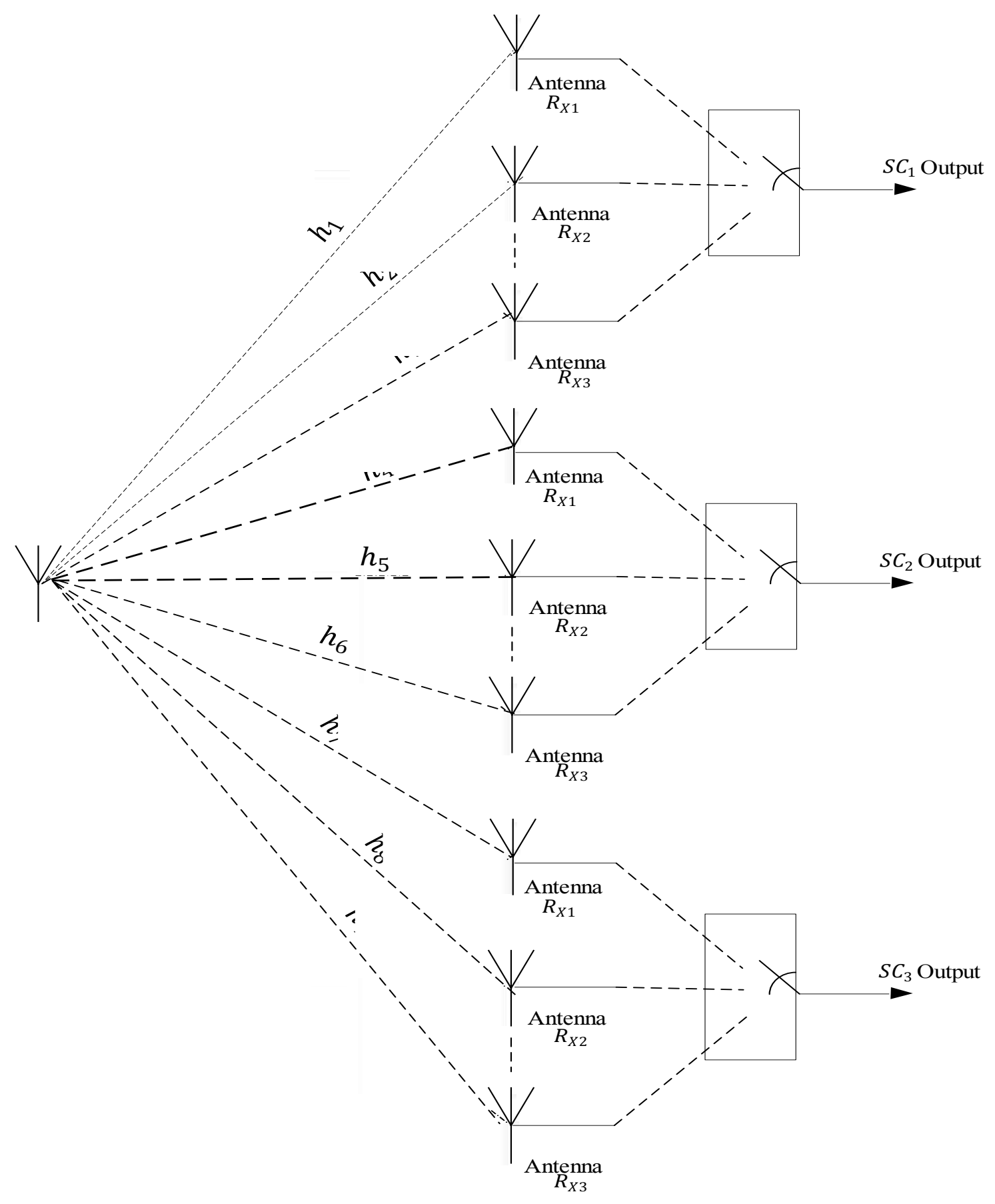

Figure 2. Block diagram of three SCs for nine receiving antenna

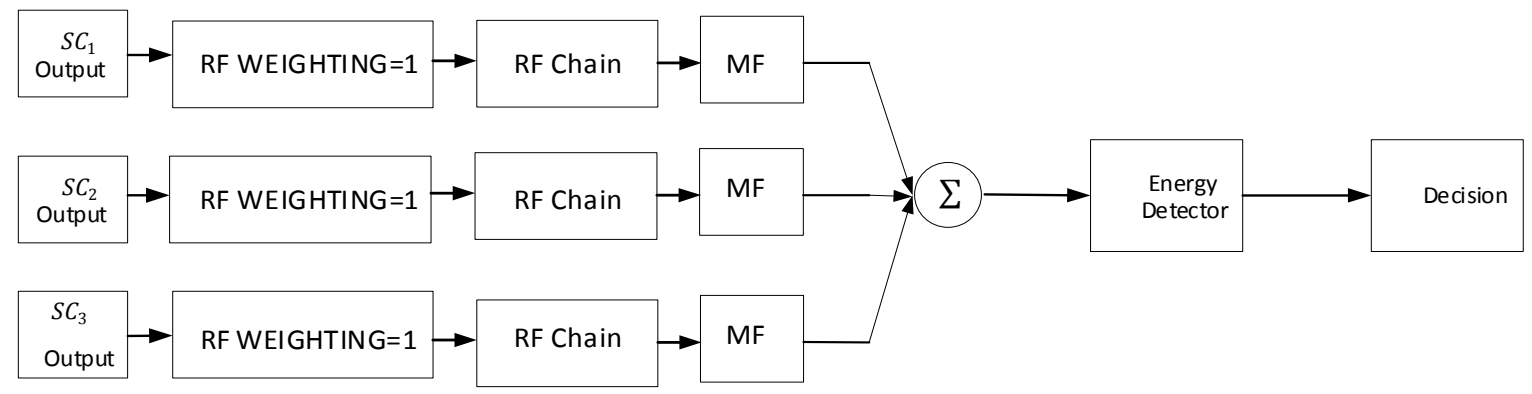

Figure 3. Block diagram of EEGC technique 
Using Equations (5) and (6), with ED and SC output SNR as the input of EGC, output signal of an enhanced EGC ' $E_{E E G C}$ ' is given as

$$
E_{E E G C}=\sum_{K=1}^{N}\left|\left(\sum_{j=1}^{N_{S C}} q_{j}(k)\right)\right|^{2} .
$$

where: $N_{S C}$ is the number of SC

$q_{j}(k)$ is the output of each SC

\section{F. False Alarm Probability of EEGC}

Using Equation (8), output of ED ' $E_{E E G C}$ ' under $H_{0}$ hypothesis is given as

$$
E_{E E G C / H_{0}}=\sum_{K=1}^{N}\left|\left(\sum_{w=1}^{N_{S C}} W_{n}(k)\right)\right|^{2} .
$$

Since $E_{E E G C / H_{0}}$ is the sum of square the distribution of test statistics becomes $\mathrm{X}^{2}$ distribution. Using $\mathrm{X}^{2}$ distribution, the output of $E D$ ' $f_{E E G C / H_{0}}(\xi)$ ' is given by $[10]$ as

$$
f_{E E G C}(\xi)=\frac{1}{\left(\sum_{K=1}^{N}\left|\left(\sum_{w=1}^{N_{S C}} \sigma_{w}^{2}(k)\right)\right|^{2}\right)^{\frac{N}{2}} \times 2^{N / 2} \mathrm{r}^{\mathrm{r}(N / 2)}} \xi^{\left({ }^{N} / 2\right)-1} \exp \left(\frac{\xi}{2 \sum_{K=1}^{N}\left|\left(\sum_{w=1}^{N_{S C}} \sigma_{w}^{2}(k)\right)\right|^{2}}\right),
$$

Equation (10) is integrated to obtain PFA of the proposed EEGC technique.

$$
\begin{aligned}
& P F A_{E E G C}=\int_{\frac{\lambda}{\left.2 \sum_{K=1}^{N} 1\left(\sum_{w=1}^{N_{S} C} \sigma_{w}^{2}(k)\right)\right|^{2}}}^{\infty} f_{E E G C}(\xi) d \xi,
\end{aligned}
$$

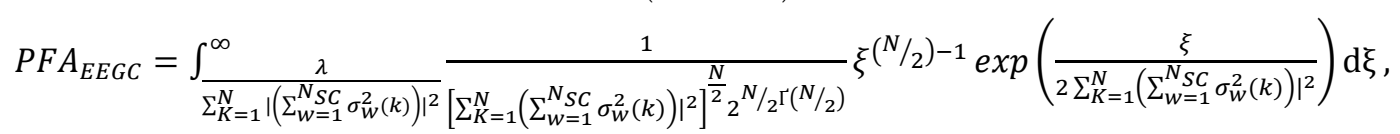

Using change of variable

$$
\begin{gathered}
t=\frac{\xi}{\left.2 \sum_{K=1}^{N}\left(\sum_{w=1}^{N_{S C}} \sigma_{w}^{2}(k)\right)\right|^{2}}, \\
\xi=2 \sum_{K=1}^{N}\left|\left(\sum_{w=1}^{N_{S C}} \sigma_{w}^{2}(k)\right)\right|^{2} t
\end{gathered}
$$

By differentiating both sides of Equation (13) gives

$$
d \xi=\left(2 \sum_{w=1}^{N} \sigma_{w}^{2}\right) d t
$$

Substituting Equations (12), (13) and (14) into (11) gives

$$
\begin{aligned}
& P F A_{E E G C}=\frac{1}{\left[\sum_{K=1}^{N}\left(\left.\left(\sum_{w=1}^{N_{S C}} \sigma_{w}^{2}(k)\right)\right|^{2}\right]^{\frac{N}{2}} 2^{N / 2} 2^{\Gamma(N / 2)}\right.} \int_{\frac{\lambda}{2 \sum_{K=1}^{N}\left(\left.\left(\sum_{w=1}^{N_{S C}} \sigma_{w}^{2}(k)\right)\right|^{2}\right.}}^{\infty}\left[2 \sum_{w=1}^{N} \sigma_{w}^{2}{ }^{(N / 2)-1} t^{(N / 2)-1} \exp (-t)\right] \times
\end{aligned}
$$

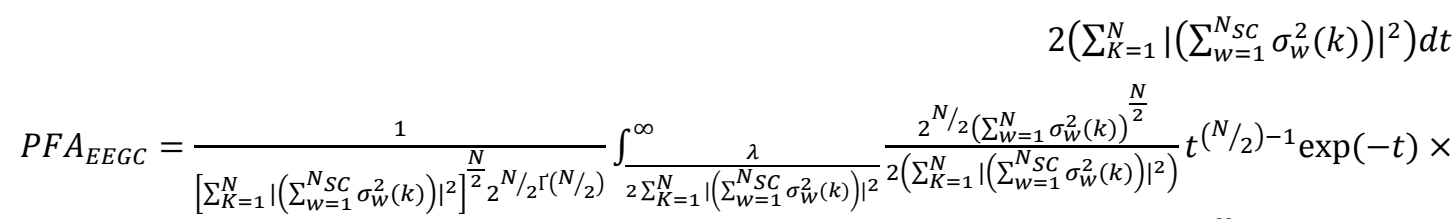

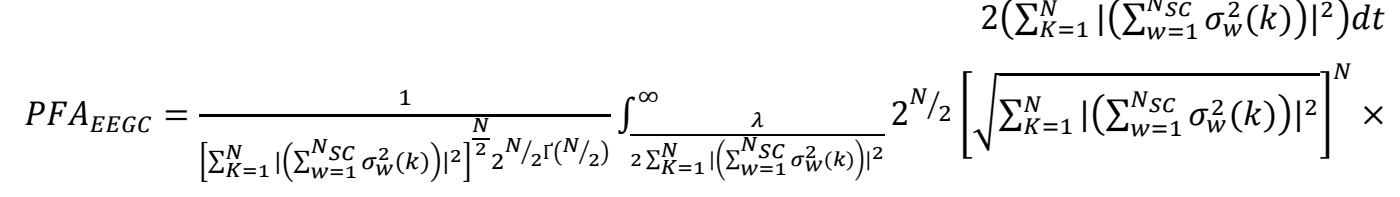

$$
\begin{aligned}
& t^{(N / 2)-1} \exp (-t) \mathrm{dt}
\end{aligned}
$$

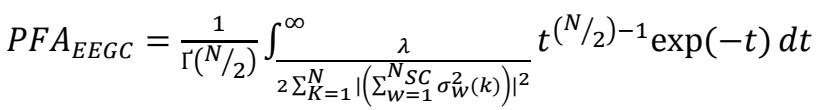

Solving Equation (15) using incomplete gamma function $\Gamma(\varepsilon, b)=\int_{\varepsilon}^{\infty} t^{b-1} \exp ^{-t} d t$, gives

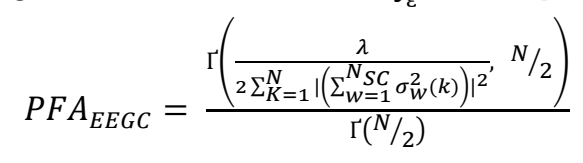

where: $\Gamma$ is the incomplete gamma function

$\lambda$ is the set threshold

$\sigma_{w}^{2}$ is the variance of noise. 
G. Probability of Detection and Probability of Missing (PM) for the Proposed EEGC

Probability of Detection (PD) for the proposed EEGC $\left(P D_{(E E G C)}\right)$ is given as

$$
P D_{(E E G C)}=\operatorname{Pr}\left(E_{E E G C}>\lambda\right) .
$$

where: $E_{E E G C}$ is the energy obtained

Probability of Missing (PM) is the probability that ULU detects the idleness of spectrum when spectrum is actually busy. PM for the EEGC $\left(P M_{(E E G C)}\right)$ is given as

$$
P M_{(E E G C)}=1-P D_{(E E G C)} \text {. }
$$

\section{Simulation Results}

Figs. 4 and 5 show the PD values obtained against SNRs for EEGC and conventional EGC at carrier frequency of $2300 \mathrm{MHz}$ with different PFAs. At PFA of 0.01, the PD values obtained at different SNRs are depicted in Fig. 4. At SNR of $4 \mathrm{~dB}$, the PD values of 0.7371 and 0.4264 are obtained for EEGC and conventional EGC, respectively, while the corresponding PD values obtained at SNR of 6 $\mathrm{dB}$ are 0.8343 and 0.5595 for EEGC and conventional EGC, respectively. Fig. 5 reveals the PD values obtained against SNR for EEGC and conventional EGC at PFA of 0.02 . The PD values obtained at SNR of $4 \mathrm{~dB}$ are 0.8090 and 0.5340 for EEGC and conventional EGC, respectively, while, 0.8348 and 0.6502 are the corresponding PD values obtained at SNR of $6 \mathrm{~dB}$. The results obtained reveal that, the proposed EEGC technique has higher detection rate than conventional EGC and this is due to selection of strongest path before applying EGC which increases signal strength of LU in the proposed technique. Also, the results obtained show that, PD increases as PFA increases, although at the expense of spectrum wastage. Figs. 6 and 7 depict PM versus SNRs for EEGC and conventional EGC at different SNRs with different PFAs. The PM results obtained at PFA of 0.01 are presented in Fig. 6. At SNR of $4 \mathrm{~dB}, \mathrm{PM}$ values of 0.2703 and 0.6007 are obtained at SNR of $4 \mathrm{~dB}$, while 0.2089 and 0.4505 are the corresponding PM values at SNR of $6 \mathrm{~dB}$. Fig. 7 shows the PM values obtained against SNR for the EEGC and conventional EGC at PFA of 0.02 . PM values of 0.2011 and 0.4860 are obtained at SNR of $4 \mathrm{~dB}$ for the EEGC and conventional EGC, respectively, while 0.1851 and 0.3598 are the corresponding PM values obtained at SNR of $6 \mathrm{~dB}$. The results obtained reveal that, the proposed EEGC has a better performance with lower PM value than the conventional EGC due to SC that is first applied to select signal with highest SNR before applying EGC. Results obtained also reveal that, PM values reduce as SNRs increase for the two techniques and this is due to performance of ED that reduces as SNR increases. The PD and PM values obtained at different SNRs are given in Tables 1 and 2 for the two values of PFA considered.

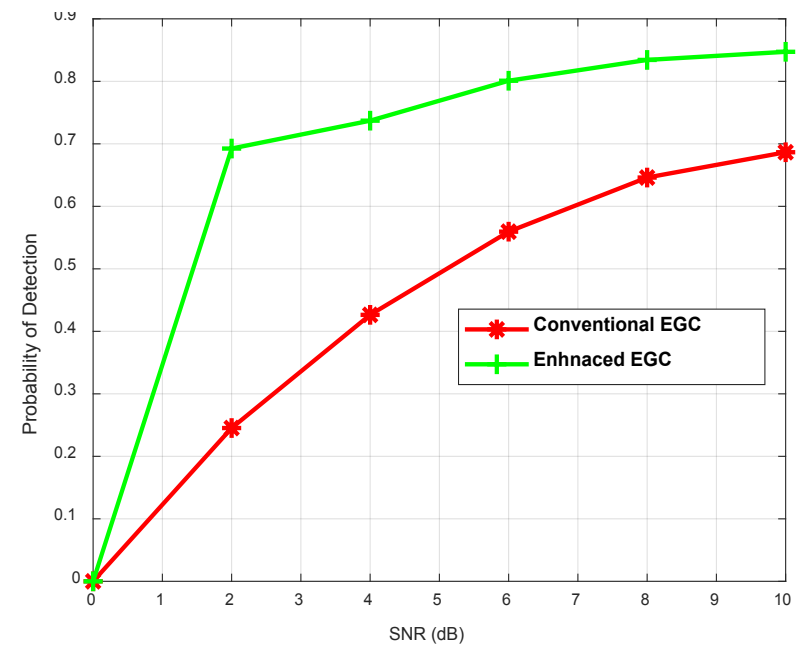

Figure 4. PD versus SNR for the enhanced and conventional EGC at PFA of 0.01

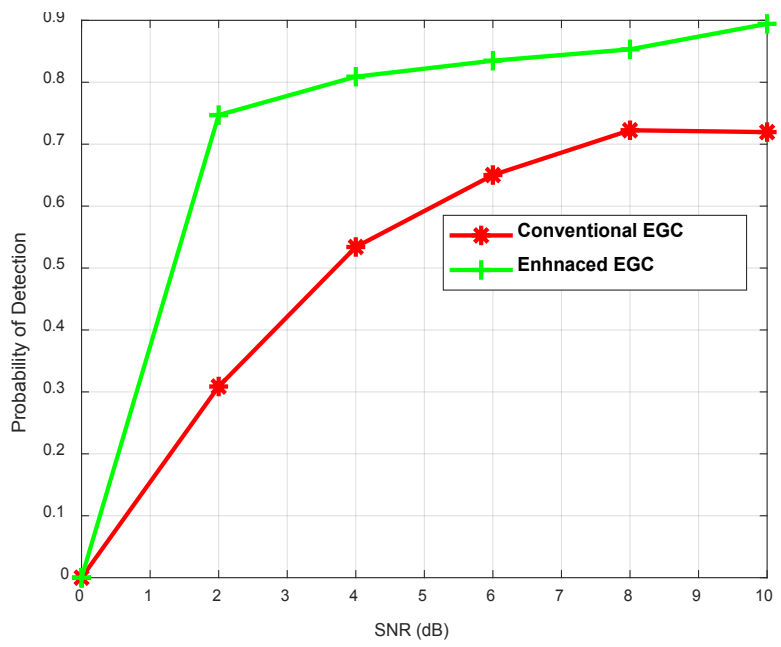

Figure 5. PD versus SNR for the enhanced and conventional EGC at PFA of 0.02

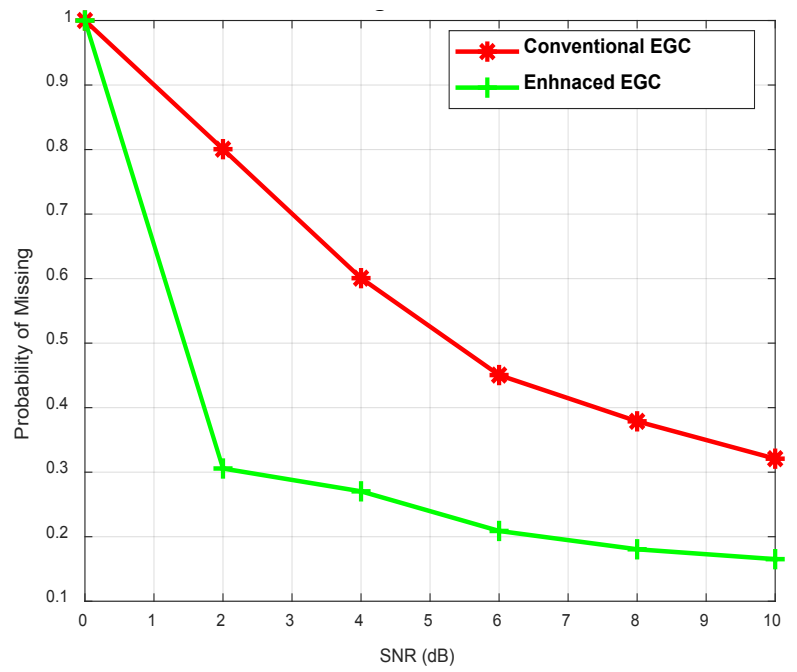

Figure 6. PM versus SNR for the enhanced and conventional EGC at PFA of 0.01 


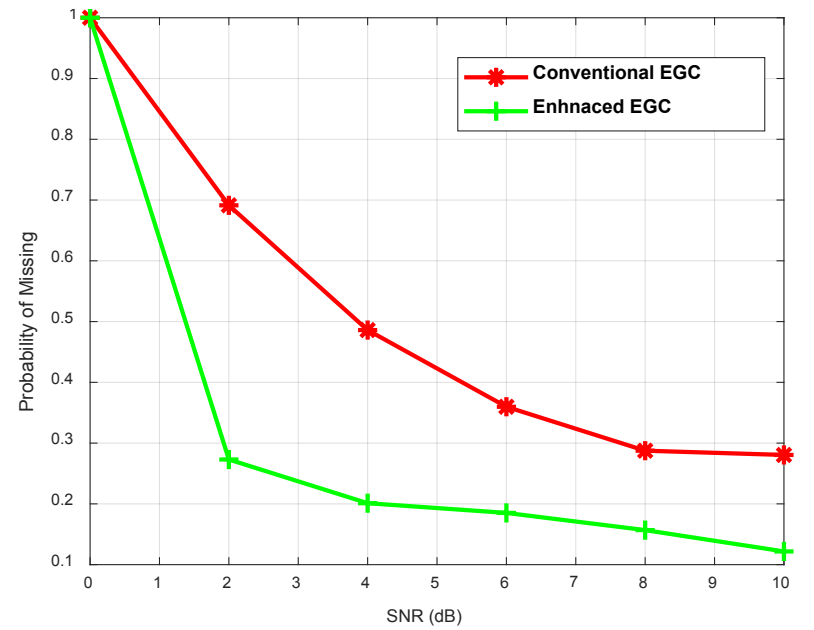

Figure 7. PM versus SNR for the enhanced and conventional EGC at PFA of 0.02

Table 1. PD and PM at PFA of 0.01

\begin{tabular}{ccccc}
\hline & \multicolumn{3}{c}{ PD } & PM \\
\hline SNR & CEGC & EEGC & CEGC & EEGC \\
0 & 0 & 0 & 1 & 1 \\
2 & 0.2453 & 0.6925 & 0.8006 & 0.3057 \\
4 & 0.4264 & 0.7371 & 0.6007 & 0.2703 \\
6 & 0.5595 & 0.8010 & 0.4505 & 0.2089 \\
8 & 0.6461 & 0.8343 & 0.3788 & 0.1805 \\
10 & 0.6866 & 0.8473 & 0.3209 & 0.1652 \\
\hline
\end{tabular}

Table 2. PD and PM at PFA of 0.02

\begin{tabular}{ccccc}
\hline \multicolumn{3}{c}{ PD } & \multicolumn{3}{c}{ PM } \\
\hline SNR & CEGC & EEGC & CEGC & EEGC \\
0 & 0 & 0 & 1 & 1 \\
2 & 0.3087 & 0.7470 & 0.6913 & 0.2730 \\
4 & 0.5340 & 0.8090 & 0.4860 & 0.2011 \\
6 & 0.6502 & 0.8348 & 0.3598 & 0.1851 \\
8 & 0.7225 & 0.8532 & 0.2875 & 0.1568 \\
10 & 0.7195 & 0.8943 & 0.2805 & 0.1217 \\
\hline
\end{tabular}

Figs. 8 and 9 depict the Sensing Time (ST) versus SNR for EEGC and conventional EGC at different PFAs. At PFA of 0.01, the ST values obtained against SNR are presented in Fig. 8. ST values of 0.3281 and $0.4006 \mathrm{~s}$ are obtained for EEGC and conventional EGC, respectively at SNR of $4 \mathrm{~dB}$, while the corresponding values of ST obtained at SNR of $6 \mathrm{~dB}$ are 0.3856 and $0.4844 \mathrm{~s}$ for EEGC and conventional EGC, respectively. Fig. 9 shows ST values obtained against SNR at PFA of 0.02. The results obtained reveal that the EEGC proposed has a lower ST values than the conventional $\mathrm{EGC}$ due to the $\mathrm{SC}$ technique employed that reduces number of RF chains and matched filters in the proposed technique. Also, the results show that ST increases with increase in SNR. Fig. 9 depicts the ST values obtained versus SNR for the EEGC and conventional EGC at PFA of 0.02 . At SNR of $4 \mathrm{~dB}$, ST values of 0.1585 and $0.2231 \mathrm{~s}$ are obtained for EEGC and conventional EGC, respectively, while 0.2184 and $0.2553 \mathrm{~s}$ are the corresponding ST values obtained at SNR of $6 \mathrm{~dB}$ for EEGC and conventional EGC, respectively.

The results obtained reveal that, ST increases as PFA increases and this is due to the fact that, the detection of ED is faster at higher threshold value than at lower threshold value. The ST values obtained for both EEGC and conventional EGC for the two PFA considered are given in Table 3 .

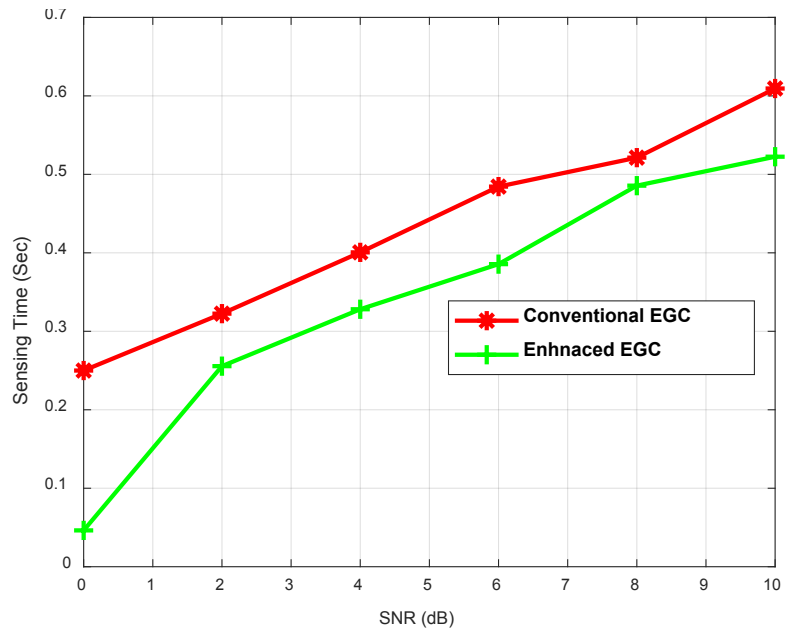

Figure 8. ST versus SNR for the enhanced and conventional EGC at PFA of 0.01

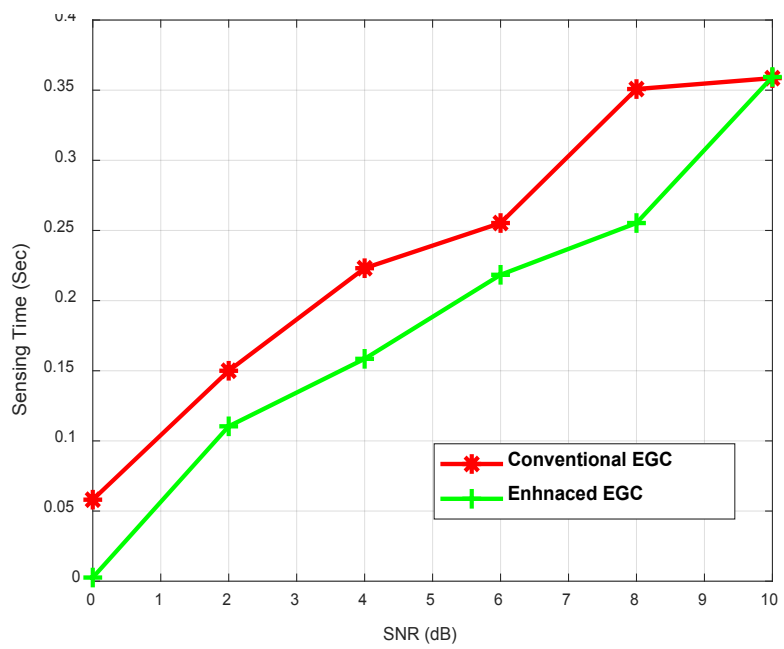

Figure 9. ST versus SNR for the enhanced and conventional EGC at PFA of 0.02 
Table 3. ST at PFA of 0.01 and 0.02

\begin{tabular}{ccccc}
\hline \multicolumn{2}{c}{ PFA $=0.01$} & \multicolumn{2}{c}{ PFA $=0.02$} \\
\hline SNR & EEGC & CEGC & EEGC & CEGC \\
\hline 0 & 0.0463 & 0.2500 & 0.0026 & 0.0581 \\
2 & 0.2556 & 0.3225 & 0.1104 & 0.1500 \\
4 & 0.3281 & 0.4006 & 0.1585 & 0.2231 \\
6 & 0.3856 & 0.4844 & 0.2184 & 0.2553 \\
8 & 0.4856 & 0.5213 & 0.2553 & 0.3508 \\
10 & 0.52250 & 0.6094 & 0.3594 & 0.3586 \\
\hline
\end{tabular}

\section{Conclusions}

In this paper, an enhanced Equal Gain Combiner for detection of spectrum in CRS is proposed. The proposed EEGC technique is incorporated with the system model and simulated using appropriate simulation parameters in line with Long Term Evolution (LTE) standard. The system is developed using three Selection Combiners (SCs), EGC and only one ED. Mathematical expression of PFA for the enhanced technique is derived using $X^{2}$ distribution which is used to set decision thresholds at 0.01 and 0.02 . The performance of the enhanced and conventional EGC has been evaluated at PFA of 0.01 and 0.02 using PD, PM and ST as performance metrics. The results show that EEGC perform relatively better than conventional EGC with lower ST, lower PM and higher PD values.

The better performance of the Enhanced EGC (EEGC) is due to selection of signal with highest SNR before applying EGC. EEGC gives lower ST values due to reduction in RF chains and matched filters of EGC compared to conventional EGC. The results of this paper show that, the proposed technique performs better as SNR increases. Therefore, the EEGC has shown a better performance than the conventional EGC.

\section{REFERENCES}

[1] Aamir, Z.S. and Talat, A. Performance Analysis of Correlated Multiple Antenna Spectrum Sensing Cognitive Radio, International Journal of Computer Applications, 2012, 5(5): 28-29.

[2] Jingbo Z., Feng R. and Da L. Maritime cognitive radio spectrum sensing based on multi-antenna cyclostationary feature detection, International Journal of Electronics, Tailor and Francis group, 2019 doi:10.1080/00207217.2019.16923 73, pp 1-17.

[3] Vadivelu, R., Sankaranyanyanan, K. and Vijayakumari, V. Matched Filter Based Spectrum Sensing for Cognitive Radio at Low Signal to Noise Ratio, Journal of Theoretical and Applied Information Technology, 2014, 62(1):108-111.

[4] Stuber, G.L. Principle of Mobile Communication, kluwer Academic, New York, 2002.
[5] Ojo, F.K. and Fagbola, F.A. Spectrum Sharing in Cognitive Radio Work Using Good put Mathematical Model for Perfect Sensing, Zero Interference and Imperfect Sensing Non-Zero interference, international Journal of Wireless Communication and Mobile Computing, 2015, 3(6): 58-59.

[6] Adeyemo, Z.K., Ojo, S.I., Abolade, R.O and Oladimeji, O.B. Modification of a Square-Law Combiner for Detection in a Cognitive Radio Network, International Journal of Wireless and Microwave Technologies, 2019, 6 (2):32-45.

[7] Pradeep, K.M., Sachin, T. and Rajeshwar, L.D. Performance Analysis of Energy Detector Matched Filter Detection and Cyclostationary Feature Detection Spectrum Sensing Techniques, International Journal of Computational Engineering Research, 2012, 2(5): 1297-1299.

[8] Adeyemo, Z.K. and Ojedokun, I.A. EGC Receiver using Single Radio Frequency Chain and Single Matched Filter over Combined Rayleigh and Rician Fading Channels, ARPN Journal of Engineering and Applied Sciences, 2014, 9(7): 992-994.

[9] Ajay, K.G., Raju, U.G., Aravind, P. and Sushma, D. Intelligent Wireless Communication System of Cognitive Radio, International Journal of Emerging Science and Engineering, 2013, 1(4): 78-84.

[10] Meenakshi, S., Prakash, C. and Nityananda, S. A brief review of cooperative spectrum sensing: issues and challenges, IEEE Transaction on Wireless Communication, 2016, 16(3):1-4.

[11] Chatziantoniou, E., Allen P. and Viandan K. Energy Detection Based Spectrum Sensing over Two-Wave with Diffuse Power Fading Channel, IEEE Transactions on radio communication. 2017.

[12] Digham, F., Aloumi M. and Simon M. Energy Detection of Unknown Signals over Fading Channel, IEEE transactions on communications, 2016, 55(1): 21-24.

[13] Sanjay, K.D., Isaac, W., Nitin, G. and Rishabh, J. (2018). Optimal Secondary Users Selection for Cooperative Spectrum Sensing in Cognitive Radio Networks, IEEE Globecom Workshop, United Arab Emirates, PP 4-9.

[14] Dong, X.J., Chen, Y. B., Yang, G.Y., Pang, X.Z. and Yang, Y. X., The Optimization of Improved Energy Detector in Cognitive Radio Network, International Conference on Computer Information Systems and Industrial Applications, Yunnan Minzu University, 2015.

[15] Haykin, S. Cognitive Radio Brain-Empowered Wireless Communications, IEEE Journal on Selected Area in Communication, 2005, 3(23): 201-210.

[16] Hano W. Gosan, N., Dongkyu, K., Sungtae, K. and Daesik, H. Advanced Sensing Techniques of Energy Detection in Cognitive Radio, Journal of Communicates and Networks, 2010, 12(1): 19-21.

[17] Mohamad, M.H. and Sani, N. M. Energy Detection Technique in Cognitive Radio System, International Journal of Engineering and Technology, 2013, 13(5): 69-74.

[18] Doha, Sonia and Ghassane. Equal Gain Combining for Cooperative Spectrum Sensing in Cognitive Radio Networks. IEEE Transactions on Wireless Communications, 2014, 10(4): 77-88. 
[19] Mittiades, C.F., David, G. and Geoge, A.R. A Comparative Performance Analysis of Interweaved and Underlay Multi-Antenna Cognitive Radio Networks, Journal of Energy supporting post-doctoral Researchers, 2013, 2(5): 68-70

[20] Taruna, T. and Bhumika P. Multiple Detectors Based Analytical Performance of Spectrum Sensing, International Journal of Advanced Computer Research, 2014, 4(1): 95-98

[21] Samrat, C. S. and Ajitsinh, N. J. Centralized Cooperative Spectrum Sensing with Energy Eetection in Cognitive Radio and optimization, IEEE International Conference on Recent Trends in Electronics Information Communication Technology,2016, India, PP 1002-1006.

[22] Garima M., Anil S. and Banerjee P. A Novel Mathematical Model for Energy Detection Based Spectrum Sensing in Cognitive Radio Networks, Wireless Personal Communicat ions, Springer, https://doi.org/10.1007/s11277-019-06783-3, 2019, pp 1-10.

[23] Rahma, B. and Hichem, B. Cooperative Spectrum Sensing for Cognitive Radio Networks: Fusion Rules Performance Analysis, IEEE Transactions on Wireless Communications, 2016, 14(2):493-496.

[24] Visser, F.E., Janssen G.J. and Pawelczak P. Multinode Spectrum Sensing Based on Energy Detection for Dynamic Spectrum Access, Conference proceeding on Vehicular Technology New York, 2008.

[25] Kaniezhil, R. and Chandraseker, C. Performance Analysis of Wireless Network with Opportunistic Spectrum Sharing via Cognitive Radio Nodes, journal of Electronic Science and Technology, 2012, 10 (4): 99-208.

[26] Nasr, A. A Noise Estimation Scheme for Blind Spectrum Sensing using EMD. Master's Theses, submitted to Electrical and Computer Engineering Department, University Alexandria, Egypt, 2017, https://scholars.unh.edu/thesis/11 08, pp 15-30
[27] Faten M., Wissam A. and Mohieddin w. A Classification of the Spectrum Sensing Techniques for Cognitive Radio, International Journal of Recent Technology and Engineering, 2019, 8(4):11586-11593.

[28] Rappaport, T.S. Wireless communications principle and practice, even publishers, Chicago, 2002.

[29] Ranjeeth, M. and Anuradha S. Cooperative Spectrum Sensing with Square Law Combining Diversity Reception, third International Conference on Signal Processing, Communication and Networking, 2015.

[30] Ramanthan V. Performance Evaluation of Equal Gain Diversity Systems in Fading Channel, Master Thesis Submitted to the Faculty of the Virgiria Polytechnic, Institute and State University, 2003.

[31] Komal, P. and Tanuja, D. Review on Spectrum Sensing in Cognitive Radio Using Multiple Antenna, International Journal of Innovative Science Engineering and Technology, 2016, 3(4):313-315.

[32] Suvarna P.J and Vaibhav S.H. Performance of Maximum Ratio Combining (MRC) MIMO Systems for Rayleigh Fading Channel, International Journal of Scientific and Research Publications, 2013, 3(2):1-3

[33] Kevin, C. Spectrum Sensing, Detection and Optimization in Cognitive Radio for Non- Stationary Primary User Signals, Ph.D Thesis submitted to Queensland University of Technology, Network and Communication, Faculty of Science and Engineering, 2012.

[34] Abolade, R.O, Ojo, S.I, Ojerinde, I.A, Adetunji, J.S, Lawal, A.T. Modification of Maximal Ratio Combining Technique for Detection of Spectrum Hole in a Cognitive Radio Network, International Journal of Wireless and Microwave Technologies, 2020, 7(2): 9-21. 